\title{
Addendum
}

\section{Chemoenzymatic Synthesis of Idesolide from Benzoic Acid}

David R. Adams, Christian Aichinger, Jonathan Collins, Uwe Rinner, Tomas Hudlicky* Synlett $2011,725$.

One author name was missing (Jonathan Collins). The current online version has been corrected. The authors apologize for this mistake. 